\title{
Clinical effect of applying high quality nursing model in nephrology department nursing administration
}

\author{
Guilan Lv ${ }^{1}$, Lixuan Huang ${ }^{2}$ \\ ${ }^{1,2}$ Nanjing Jinling Hospital, Nanjing Jiangsu, 210002, China
}

\begin{abstract}
Keywords: Nephrology department, Nursing management, High-quality nursing model, Nephrotic
\end{abstract} patient.

\begin{abstract}
Objective: explore the clinical effect of implementing high-quality nursing model in patient's nursing administration work in nephrology department. Method: select 130 cases of patients received in in nephrology department of our hospital during Feb of 2015 to Feb 2016 as research object, divided into two teams based on the hospitalization sequence, reference group (admission time Feb 2015- Aug 2015) and observation group (admission time Sep 2015-Feb 2016), 65 cases in each group for routine nursing, and high-quality nursing. Evaluate the psychologic status of two groups of patients before and after nursing, and observe the occurrence of nurse-patient disputes and complaints. Result: there is no notable difference between SAS and SDS score of two groups of patients before nursing ( $\mathrm{P}>0.05)$; after nursing, SAS and SDS score in observation group is obviously higher than reference group, with significant difference $(\mathrm{P}<0.05)$; No nurse-patient disputes and complaints happen in observation group but there are 10 cases in the reference group, 10 cases of nurse-patient disputes (15.38\%) and 4 cases of complaints (6.15\%). Comparing two groups, difference has statistical significance $(\mathrm{P}<0.05)$ Conclusion: conducting high-quality nursing model on nephrotic patients can effectively improve patients' anxiety and depression, and relieve nurse-patient relationship, reduce the occurrence of nurse-patient disputes and complaints, and deserves widely clinic application.
\end{abstract}

\section{Introduction}

Nephropathy is a common clinical internal medicine disease with complex condition and various types. If the condition of nephrotic patient's condition cannot be controlled very well in the early stage, it is extremely likely to develop into uremia or other more serious disease, brining bad influence to patients mentally and physically [1]. Besides, mental stress resulted from the disease is harmful for the patients' prognosis. With the improvement of people's living standard, their demands for medical nursing service is higher and higher. Thus except for reasonable medical methods, scientific nursing intervention measure is the effective method in clinic treatment for disease control. Hospital should pay attention to the nephrotic patient's nursing work. This article applies high-quality nursing intervention in some nephrotic patients' nursing administration for interference with good effect achieved to provide reliable basis for future clinical work. Now the report is as below:

\section{Data and methods}

\section{General data}

This research selects totally 130 cases of nephrotic patients in our hospital as the research object all receiving treatment during Feb 2015 to Feb 2016, in accordance with the clinic related nephrotic disease diagnosis standard [2]. Take 65 cases of patient hospitalized during Feb 2015 to Aug 2015 as the reference group, and 65 cases hospitalized during Sep 2015 to Feb 2016 as the observation group. 
The male-female proportion in reference group is 35:30, age scope of 31-65 years old, average age of 45.69 \pm 2.48 years old; 15 cases of chronic glomerulonephritis, 17 cases of nephrotic syndrome, 10 cases of kidney failure, 13 cases of renal insufficiency, 10 cases of others. The male-female proportion in the observation group is 34: 31, age scope of 30-65 years old, average age of 45.22 \pm 2.39 years old, 14 cases of chronic glomerulonephritis, 18 cases of hydremic nephritis, 11 cases of kidney failure, 14 cases of renal insufficiency, 8 cases of others. After comparing the gender proportion, age scope, disease type and other general data in two groups, we find there is no obvious difference $(\mathrm{P}>0.05)$, and there is the compatibility.

\section{Nursing method}

\section{Reference group}

Conduct patients in this group with routine nursing intervention such as keeping close eyes on patients' illness change, inspecting their consciousness, urine amount and other index, and asking patients to take quantitative medicines on time, keep the ward environment clean and tidy, and instructing patients and families with disease related knowledge.

\section{Observation group}

Provide patients in this group with high-quality nursing mainly including environment nursing, popularizing disease knowledge, mental relaxation training, drug use and other contents. Specific implementation measurements are as below:

Environment nursing: after getting into the hospital, nursing staffs should make self-introduction in a friendly and kind attitude, and then introduce the doctor in charge, and basic information in this department and other patients in this ward so as to relieve their strangeness of firstly admission into the hospital. Establish health record for patients, and record their height, weight, disease type, attached medicines and other details so as to understand each patient's condition and set patients with special disease into the same ward. Remind patients not smoking and making confused noises in the ward. Clean the patient ward every day, open window for ventilation to keep air circulation so as to provide a peaceful and comfortable treatment environment. Encourage patients' actively to communicate and exchange so that they stay in a free and relaxed atmosphere during their hospitalization.

Popularization of disease knowledge: Due to differences in patient age, personality characteristics, standard of culture, and disease severity, they have different understanding of their own disease. Nursing staffs should have a clear awareness of this point and instruct them with the disease reason, clinic features, treatment time, and the effect of treatment medicines based on their practical condition so as to help them to comprehensively understand their own disease. For questions raised by patients, nursing staffs should use easy and simple words to answer. Meanwhile, explain the relationship between life factors such as diet and emotional control with the disease treatment effect to patients, and get patients with the same disease together through the way of playing videos and picture-text lecture to enlarge patients' understanding of diseases. During the process of popularizing the knowledge, nursing staffs and patients have more communication chances, which is good for establishing good relationship.

Mental relaxation training: due to suffering from the disease pain and worry about medical expenses, some patients have anxious and depressed emotions, which will influence the treatment effect and more seriously some patients' condition will worsen and increase treatment difficulties. Thus, nephrotic patients' mental relaxation training is necessary during their therapy. Nursing staffs can play gentle and soft music instructing patients to close eyes for meditation and adjust breath in accordance with the music rhythm so as to recover good mood, or instruct patients to lie flatly on the bed with left and right hand putting in the belly and chest, lifting to the highest point through strong breathing and holding breath for three seconds and then exhaling slowly. Abdominal respiration training should be conducted for ten mins every day, once in the morning and evening. It is worth noticing that nursing staffs should remind patients that the time between breathing in and out should be proportion of 1:1. In this way, patients can achieve a state of relaxation. Instruct patients to lie on 
the bed for muscle shrinkage and diastole in the sequence from face, neck, chest, and belly to the back, lasting ten mins each time, once in the morning and evening. This kind of relaxation method can help relax the tense muscle and release bad mood. Nursing staffs can also actively communicate with patients when touring the ward asking whether they have discomforts and encourage them to speak out their feelings.

Drug use instruction: it must be prudent in drug use for nephrotic patient. Try less or try to avoid drugs with strong renal toxicity. If needed for patients' condition, the amount should controlled. Nursing staffs must be clear about the mutual effect between different drugs to prevent the renal toxicity worsening due to wrong compatibility of medicines, sedative hypnotics, anti-hypertension medicine, tetracycline antibiotics and antidepressants are drugs used with caution. Patients with normal liver function but renal insufficiency should choose liver and kidney excretory drugs for treatment so as to reduce the burden to the patients' kidney, and reduce the occurrence of acute kidney failure, glomerulus dysfunction and other combined disease.

\section{Observation index}

The nursing intervention time of two groups should last for one month. Use SAS and SDS to judge the mental change of patients in two groups. If the scores in SAS and SDS are higher than 50, it shows that the patient is with depression and anxiety. The more scores, the severity of depression and anxiety. Observe and record the occurrence of nurse-patient disputes and complaints during the nursing process and compare the occurrence.

\section{Statistical treatment}

Apply SPSS20.0 statistics software to analyze the data acquired in this research, enumeration data shown as \%, for $x^{2}$ test, measurement data shown as $\bar{x} \pm s$, tested by t. If the test result is $\mathrm{P}<0.05$, it proves that the difference between group data is notable.

\section{Result}

\section{Comparison of SAS and SDS scores in two groups before and after nursing}

If patients in two groups have more than 50 scores in SAS and SDS before nursing, it shows that patients in two groups have depression and anxiety emotion, the difference has no statistical significance $(\mathrm{P}>0.05)$. After corresponding nursing intervention, SAS and SDS scores of patients in two groups have declined, which shows that patients' depression and anxiety mood has been improved. But SAS and SDS scores in observation group is obviously lower than the reference group, with significant difference $(\mathrm{P}<0.05)$. Specific details shown as the Table 1 .

Table 1. Comparison of SAS and SDS scores of patients in two groups before and after nursing ( $\bar{X} \pm S$, score)

\begin{tabular}{ccccc}
\hline Group & Number of case & Time & SAS & SDS \\
\hline Reference & \multirow{2}{*}{65} & Before nursing & $60.34 \pm 3.61$ & $58.67 \pm 3.55$ \\
group & & After nursing & $55.62 \pm 1.77^{\triangle}$ & $54.92 \pm 1.04^{\triangle}$ \\
Observation & \multirow{2}{*}{65} & Before nursing & $60.48 \pm 2.94$ & $59.11 \pm 3.67$ \\
group & & After nursing & $49.68 \pm 1.71^{\triangle}$ & $49.26 \pm 1.15^{\triangle}$ \\
\hline
\end{tabular}

Notes: comparison inside group, $\mathrm{P}<0.05$; comparison between two groups: $\mathrm{P}<0.05$, difference has statistical significance.

\section{Comparison of the occurrence of nurse-patient disputes and complaints in two groups during the nursing process}

The occurrence of nurse-patient disputes and complaints in reference group during the nursing process is obviously higher than the observation group with significant difference $(\mathrm{P}<0.05)$. Details shown in Table 2: 
Table 2. Comparison of the occurrence of nurse-patient disputes and complaints in two groups during the nursing process (n/\%)

\begin{tabular}{cccc}
\hline Group & Number of case & Nurse-patient disputes & Complaint issue \\
\hline Reference group & 65 & $10(15.38)$ & $4(6.15)$ \\
Observation group & 65 & $0(0.00)$ & $0(0.00)$ \\
x2 value & & 10.83 & 4.13 \\
P value & & 0.01 & 0.04 \\
\hline
\end{tabular}

\section{Discussion}

Currently, people's understanding of health concept is improving, thus the hospitalized patients' demand for therapy and nursing work is changing from singularity to diversity. Therefore, regular nursing model cannot satisfy patient's demand and a lot of medical disputes have been caused due to this. Related researches show that, if the nursing level in the hospital is high, it will promote the patients to improve the patient's feeling of comfort and mental state so as to reduce the nurse-patient disputes and complaints issues [4].

High-quality nursing is a model advocating to care for patient's mental health from interior to exterior with its clinic application value recognized already. This article uses two groups of routine nursing and high-quality nursing to intervene the nursing of patients with nephrotic disease and then proves this view point through comparing the nursing effect. In this research, high-quality nursing implemented in patients in observation group mainly include environment nursing, popularization of disease knowledge, mental relaxation training and drug and other intervention measurements. Environment nursing can help patients stay in a comfortable and tidy environment for therapy as well as can reduce the occurrence of combine diseases happening due to bacterial infection. The popularization of disease knowledge can not only enlarge patient's understanding of disease, but can also promote to establish good relationship between nursing staffs and patients so as to effectively avoid the occurrence of nurse-patient disputes and complaints[6]. Result of this research shows that there is no occurrence of nurse-patient disputes and complaints in observation group but 10 cased of nurse-patient disputes and 4 cases of complaints. Difference in two groups is significant $(\mathrm{P}<0.05)$, which has impact the normal implementation of therapy and nursing work, harmful for patient's recovery. Nephrotic patients are inevitably under pressure mentally and physically, and the mental stress will influence the patient's condition. Thus, proper relaxation is a method helpful for improving patients' disease [7]. In this research, SAS and SDS scores of patients in observation group after nursing is obviously lower than the reference group $(\mathrm{P}<0.05)$. Compared with routine nursing, conducting high-quality nursing on nephrotic patients can achieve ideal clinic effect not only releasing patients' bad mentality but also improving nurse-patient relationship [8].

To sum up, during the nephrotic patients nursing administration, applying high-quality nursing model has good effect on patients' depression and anxiety mood and improving nurse-patient relationship with high clinic application value.

\section{Reference}

[1] Shen Xiaoqin. Evaluation of the effectiveness of applying high-quality nursing administration model in nephrology department nursing management, The World Clinical Medicine,2015,9(07):1.

[2] Gao Yiqing. Application and effect of high-quality nursing service in nephrology, Medical Information,2015,28(26):160.

[3] Tan Caihong. Discussion on the influence of high-quality nursing service on patient's depression and anxiety hospitalized in nephrology department, Contemporary Medicine,2014,20(10):10-108. 
[4] Wang Caiyun, Zhang Ruiyun. Observation of effect of applying high-quality nursing service to improving patients' negative emotions in nephrology department, Modern Chinese Doctor,2016,54(08):147-149.

[5] Zhuang Zhaohong. Analysis of the application of high-quality nursing service in improving patients' negative emotions in nephrology department, Guide of China Medicine,2015,13(18):211-212.

[6] Yang Jinli. Discussion on method applying high-quality nursing service to 68 cases of patients in nephrology department, Healthy People, 2016,10(04):258.

[7] Ji Minghui. Application value of high-quality nursing intervention in hemodialysis of patients with diabetic nephropathy, World Latest Medicine Information,2016,16(19):176-177.

[8] Peng Shuijuan. Implementation experience and effect of carrying out high-quality nursing service in nephrology department ward, The Journal of Medical Theory and Practice,2014,27(06):814-815. 